\title{
Serratia plymuthica
}

National Cancer Institute

\section{Source}

National Cancer Institute. Serratia plymuthica. NCI Thesaurus. Code C86740.

A species of facultatively anaerobic, Gram negative, rod shaped bacteria in the phylum Proteobacteria. This species is motile, peritrichous, esterase positive, decarboxylase and citrase negative and can support growth on and produce acid from arabinose, melibiose, sorbitol, and xylose. S. plymuthica is found in the soils surrounding the root of various plants and in the water supply. It is a pathogen that can cause infections including osteomyelitis, peritonitis, pneumonia, sepsis and wound infections. 